\title{
Structure Factors and Their Distributions in Driven Two-Species Models
}

\author{
G. Korniss* and B. Schmittmann \\ Center for Stochastic Processes in Science and Engineering and Department of Physics, \\ Virginia Polytechnic Institute and State University, Blacksburg, Virginia 24061-0435
}

(June 12, 1997)

\begin{abstract}
We study spatial correlations and structure factors in a three-state stochastic lattice gas, consisting of holes and two oppositely "charged" species of particles, subject to an "electric" field at zero total charge. The dynamics consists of two nearest-neighbor exchange processes, occuring on different times scales, namely, particle-hole and particle-particle exchanges. Using both, Langevin equations and Monte Carlo simulations, we study the steady-state structure factors and correlation functions in the disordered phase, where density profiles are homogeneous. In contrast to equilibrium systems, the average structure factors here show a discontinuity singularity at the origin. The associated spatial correlation functions exhibit intricate crossovers between exponential decays and power laws of different kinds. The full probability distributions of the structure factors are universal asymmetric exponential distributions.
\end{abstract}

\section{INTRODUCTION}

The study of correlations and structure factors provides a sensitive probe into the characteristics of collective behavior in many-particle systems. For example, in a system with short range microscopic interactions, maintained in thermal equilibrium, spatial correlations will in general decay exponentially. Long-range spatial correlations, characterized by power laws, are observed only if the system is at a critical point. In contrast, when such systems are driven into non-equilibrium steady states, long-range correlations are often present in large regions of the phase diagram [1]. A model system in which such anomalous correlations are easily observed and studied is the driven Ising lattice gas (the "standard model") [2,5]. The drive ("external field") biases particle jumps along a specific lattice axis, thus forcing the system into a non-equilibrium steady state. One of the most intriguing and unexpected features of this system is the presence of long-range spatial correlations at all temperatures above criticality, due to the breakdown

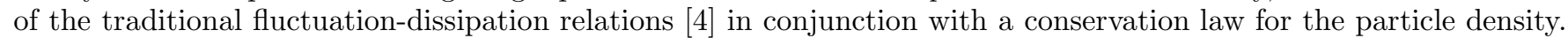
In momentum space, the structure factor develops a discontinuity singularity at the origin [5].

In experiments, correlations are typically studied by photon, electron or neutron beam scattering techniques. The scattering intensity is closely related to the structure factor. Depending on the actual physical system, this quantity is the Fourier transform of the "density-density" correlations, where, e.g., in a ferromagnetic system "density" stands for the local magnetization. Even in the stationary case, i.e., when the averages are not expected to be time-dependent, the densities themselves are fluctuating quantities in both space and time. Thus, when data are taken, it is crucial to compare the time scale of these fluctuations to the duration of a typical "snap-shot". If the former is much shorter than the sampling time interval, then even one measurement practically results in a temporal average. In this case, the scattering intensity is a direct measure of the average structure factor. In the opposite scenario each individual snap shot appears as a random pattern of speckles. The collection of many snap shots, however, represents the full distribution of the fluctuating density-density products. This phenomenon has long been known in laser scattering experiments and the statistical properties of the random speckles are well established [6]. Using Monte Carlo simulations, it is particularly easy to probe fluctuating quantities in terms of the their distributions: each measurement corresponds to one configuration at a certain instant of time so that there are no "experimental" difficulties in achieving fine sampling. Since driven lattice gases exhibit generically singular density-density correlations, a study of the associated distributions is expected to be particularly interesting.

In this paper, we will focus on a generalization of the standard model, similar in spirit to the one leading from the Ising model to spin-1 [7] or Potts [8] models. Instead of just a single species of particles, we now consider two (labelled as +'s and -'s) which are driven in opposite directions, subject to periodic boundary conditions. Empty lattice sites

\footnotetext{
*Permanent address: Supercomputer Computations Research Institute, Florida State University, Tallahassee, Florida 32306-4052
} 
are referred to as holes. This generalization is motivated by a variety of physical systems, ranging from fast ionic conductors with several mobile ion species [9] and water droplets in microemulsions with distinct charges [10] to gel electrophoresis 11] and traffic flow [12]. For simplicity, we neglect the usual Ising nearest neighbor interaction and retain "only" the excluded volume constraint. The model thus corresponds to the high-temperature, large-drive limit of a more complicated interacting system.

This multi-species model, in both one and two dimensions, has been studied in detail [12 18]. In its simplest version, particles are allowed to exchange with holes only. Monte Carlo simulations [13] in two dimensions and mean-field studies [14,15] show that there is a transition, controlled by particle density and drive, from a spatially homogeneous (disordered) phase to a charge segregated one, where the excluded volume constraint leads to the mutual blocking of particles. In this paper we extend our previous studies on structure factors [19] and their distributions 20] to the more general case where the particles are also allowed to exchange amongst themselves: we "soften" the excluded volume constraint by allowing exchanges of nearest neighbor, oppositely charged particles on a time scale $\gamma$ which is distinct from that of the particle-hole exchange [16]. Here, the blocking transition still occurs, as part of a more complex phase diagram [16]. A particularly interesting case emerges when the two time scales are chosen to be equal, i.e., $\gamma=1$ : here, equal charges are completely uncorrelated (up to trivial finite-size effects) while "hidden", non-trivial correlations survive between opposite charges. We add that this model in one dimension, at infinite drive and for arbitrary $\gamma$, has been solved exactly by Godrèche and Sandow [18].

We will focus on the disordered phase of the system, where we have a sound analytic understanding of the dynamics in terms of Langevin equations. We will study not only the averages but also the full distributions of the steady-state structure factors, using Monte Carlo simulations and a continuum field theory. Finding excellent agreement between our simulations and analytic results, we can trace the characteristics of the distributions back to the structure of the underlying Langevin equations. Given these relations, measurements of structure factor distributions in real systems should provide considerable information about the associated dynamics.

The paper is organized as follows. In the next section, we define the microscopic model and give some details of the simulations. In Section III, we introduce the underlying Langevin equations and calculate the average structure factors, the corresponding spatial correlations, and the theoretical distributions of the structure factors. In the final section, we discuss our results and conclude with a brief summary.

\section{THE MICROSCOPIC MODEL}

We consider a two dimensional fully periodic lattice with $L \times L$ sites, each of which can be empty or occupied by a single particle. To account for the presence of two species, we introduce two occupation numbers $n_{\mathbf{x}}^{+}$and $n_{\mathbf{x}}^{-}$, with $n$ being 0 or 1 , depending on whether a positive or negative particle is present at site $\mathbf{x}$. The excluded volume constraint implies $n_{\mathbf{x}}^{+} n_{\mathbf{x}}^{-}=0$, for any $\mathbf{x}$. To model the system at zero total charge, we choose $\sum_{\mathbf{x}}\left[n_{\mathbf{x}}^{+}-n_{\mathbf{x}}^{-}\right]=0$, i.e., the average densities of positive and negative particles are the same:

$$
\bar{\rho}=\frac{1}{L^{2}} \sum_{\mathbf{x}} n_{\mathbf{x}}^{+}=\frac{1}{L^{2}} \sum_{\mathbf{x}} n_{\mathbf{x}}^{-},
$$

Since the dynamics conserves both densities separately, $\bar{\rho}$ is a constant. In the absence of the drive, the two species of particles are distinguished only by their label: both types hop randomly to nearest-neighbor empty sites, with the same rate $\Gamma$. In addition, nearest neighbor pairs of opposite charges exchange with a rate $\gamma \Gamma$. The external drive is directed along a specific lattice axis, labelled as the $+x_{\|}$-direction. Reminiscent of a uniform "electric" field $E$, it exponentially suppresses jumps against the force. Specifically, during one Monte Carlo step $2 L^{2}$ nearest-neighbor bonds are selected at random. If a particle-hole pair is encountered, an exchange takes place with probability

$$
W_{p h}=\Gamma \min \left\{1, \exp \left(q E \delta x_{\|}\right)\right\}
$$

where $q= \pm 1$ is the charge of the particle and $\delta x_{\|}= \pm 1,0$ is the change of the $x_{\|}$coordinate of the particle due to the jump. Similarly, if the neighboring sites are occupied by opposite charges, a particle-particle exchange (or charge transfer) is attempted with probability

$$
W_{p p}=\gamma \Gamma \min \left\{1, \exp \left(E \delta x_{\|}\right)\right\}
$$

where now $\delta x_{\|}$is the change in the $x_{\|}$coordinate of the positive particle due to the jump. Note that we do not introduce a factor of 2 in the exponential here, as one might have expected for a real electric field. This choice leads to a simpler Langevin equation without significantly affecting the phase diagram. Needless to say, it is irrelevant whether exchange takes place or not, if both sites carry identical content. 
For our simulations, we set $\Gamma=1$, so the control parameters are $\bar{\rho}, E$ and $\gamma$. On lattices with $L$ ranging from 30 to 100 , the system is initialized with random configurations of various particle densities. Runs last from $2.5 \times 10^{5}$ to $5 \times 10^{5}$ MCS. The first 62500 MCS are discarded to allow the system to settle into steady state. Then, we measure the Fourier transforms of $n_{\mathbf{x}}^{ \pm}$every $125 \mathrm{MCS}$, defining them in the usual way:

$$
n_{\mathbf{k}}^{ \pm}=\sum_{\mathbf{x}} e^{-i \mathbf{k x}} n_{\mathbf{x}}^{ \pm} .
$$

In the following, we will investigate equal-time density-density operators in momentum space, considering both their full distributions as well as their (ensemble or time) averages. In the literature, the term "structure factor" typically refers to the averages, i.e.,

$$
S^{\alpha \beta}(\mathbf{k}) \equiv \frac{1}{V}\left\langle n_{\mathbf{k}}^{\alpha} n_{-\mathbf{k}}^{\beta}\right\rangle,
$$

where $\alpha, \beta=+,-; \mathbf{k}=\frac{2 \pi}{L}\left(m_{\perp}, m_{\|}\right) \neq \mathbf{0}$ and $V=L^{2}$ is the volume. Occasionally, especially when discussing the full distributions, we will use the word "structure factor" for the fluctuating two-point operator itself. In the disordered phase, $S^{\alpha \beta}$ is the Fourier transform of the usual equal-time correlation function

$$
G^{\alpha \beta}(\mathbf{x}) \equiv\left\langle n_{\mathbf{x}}^{\alpha} n_{\mathbf{o}}^{\beta}\right\rangle-\left\langle n_{\mathbf{x}}^{\alpha}\right\rangle\left\langle n_{\mathbf{o}}^{\beta}\right\rangle
$$

Thus, if $G$ is even in $\mathbf{x}, S$ will be real, so that an imaginary part of $S$ signals a part of $G$ which is odd in $\mathbf{x}$. By charge symmetry, we expect $G^{++}=G^{--}$. Clearly, both must be even in x, so that the associated $S$ 's are real. On other hand, we have

$$
G^{+-}(\mathbf{x}, E)=G^{+-}\left(x_{\perp},-x_{\|},-E\right)
$$

in the presence of the drive so that $S^{+-}$may have an imaginary part (which must be odd in $\left.E\right)$. Finally, $G^{-+}(\mathbf{x})=$ $G^{+-}(-\mathbf{x})$ follows from $(6)$ by translation invariance. Turning to the full distributions, these can be constructed from the time series of $\frac{n_{\mathbf{k}}^{+} n_{-\mathbf{k}}^{+}}{V}, \frac{\operatorname{Re}\left[n_{\mathbf{k}}^{+} n_{-\mathbf{k}}^{-}\right]}{V}$ and $\frac{\operatorname{Im}\left[n_{\mathbf{k}}^{+} n_{-\mathbf{k}}^{-}\right]}{V}$ in the steady state. Exploiting symmetries again, we note that $\frac{n_{\mathbf{k}}^{+} n_{-\mathbf{k}}^{+}}{V}$ and $\frac{n_{\mathbf{k}}^{-} n_{-\mathbf{k}}^{-}}{V}$ are distributed identically, so that we need to consider only the former. Further, only $\frac{n_{\mathbf{k}}^{+} n_{-\mathbf{k}}^{+}}{V}$ and $\frac{n_{\mathbf{k}}^{-} n_{-\mathbf{k}}^{-}}{V}$ are necessarily real, while $\frac{n_{\mathbf{k}}^{+} n_{-\mathbf{k}}^{-}}{V}$ will generically be complex.

We simulate systems with $\gamma$ ranging from 0 to 1 . For small $\gamma$ 's we choose $E$ and the density $\bar{\rho}$ in such a way that the system is in the homogeneous phase. For larger $\gamma^{\prime}$ s $\left(\gamma>\gamma_{c} \simeq 0.62\right)$ the charge exchange mechanism suppresses the ordered phase entirely [16] so we can pick arbitrarily large fields at any density. A particularly interesting case occurs for $\gamma=1$. Here, the rates for particle-hole and particle-particle exchanges become equal, i.e., $W_{p p}=W_{p h}$, so that a positive (negative) particle can no longer distinguish a negative (positive) one from a hole. Thus, a positive (negative) particle experiences biased diffusion, slowed only by encounters with other positive (negative) particles, just as in the case of a single, non-interacting species, whose steady state probability distribution of configurations (i.e., the steady state solution of the associated master equation) is exactly known to be uniform [21]. For our case, this implies that the marginal distribution of the occupation numbers of one species is uniform, i.e., $P\left[\left\{n_{\mathbf{x}}^{ \pm}\right\}\right]=\sum_{\left\{n_{\mathbf{x}}^{\mp}\right\}} P\left[\left\{n_{\mathbf{x}}^{+}, n_{\mathbf{x}}^{-}\right\}\right] \propto 1$. Thus, we expect questions regarding only one species of particles to have trivial answers, e.g. $G^{++}(\mathbf{x})$ must vanish for $\mathbf{x} \neq \mathbf{0}$ in an infinite system or yield the finite-size fluctuations in a finite one. On the other hand, the two-point function between opposite charges can display interesting structures, e.g. long-range correlations, as a result of the full distribution $P\left[\left\{n_{\mathbf{x}}^{+}, n_{\mathbf{x}}^{-}\right\}\right]$not being uniform. We note briefly that a completely "flat" steady state, $P\left[\left\{n_{\mathbf{x}}^{+}, n_{\mathbf{x}}^{-}\right\}\right] \propto 1$, is obtained for $\gamma=2$, as in the one-dimensional version of our model [18].

In Fig. 1, we present the results for the three independent $S$ 's found in the $100 \times 100$ system at a small value of $\gamma$ and note the discontinuity singularity of these objects at the origin. In Fig. 2, we show the same quantities for $\gamma=1$ and draw special attention to the fact that, while $S^{++}$does not depend on $\mathbf{k}$ at all, $S^{+-}$exhibits a highly nontrivial k-dependence. Fig. 3 and 4 present the structure factor distributions for the smallest longitudinal and transverse wave vectors, respectively. Before discussing the data in detail, we will first present the theoretical framework within which they can be understood. In particular, we will focus on two points, namely first, the emergence of discontinuity singularities in the structure factors at $\mathbf{k}=\mathbf{0}$, and their consequences for long-range correlations in real space, and second, the origin of the asymmetric exponential form of the distributions. This will then be followed by a comparison between our theoretical predictions and the simulations. 


\section{COARSE-GRAINED DESCRIPTION}

To extract the behavior at large distances (or small $k$ in momentum space), a continuum field theory for the slow variables of the model is most appropriate. To find such a description, we must (i) identify the slow variables of the theory, and (ii) obtain a set of equations of motion for these quantities, corresponding to a coarse-grained version of the microscopic dynamics. For generality, we consider the $d$-dimensional case when $\mathbf{x}_{\|}$is directed along the electric field and $\mathbf{x}_{\perp}$ is in the $(d-1)$-dimensional subspace, perpendicular to the field. Time is denoted by $t$. Then, the slow variables are easily identified as the conserved densities, $\rho^{ \pm}(\mathbf{x}, t)$, of the two species. The most systematic way to arrive at their equations of motion is to perform an $\Omega$-expansion [22,23]: After partitioning the whole system into sufficiently large blocks of size $\Omega$, one splits the particle densities associated with the block centered at $\mathbf{x}$ into a macroscopic part $\left(\rho^{ \pm}\right)$and a fluctuating one $\left(\chi^{ \pm}\right)$:

$$
\frac{1}{\Omega} \sum_{\mathbf{x}^{\prime} \in \mathrm{b}(\mathbf{x})} n_{\mathbf{x}^{\prime}}^{ \pm}=\rho^{ \pm}(\mathbf{x}, t)+\Omega^{-1 / 2} \chi^{ \pm}(\mathbf{x}, t)
$$

This decomposition is inserted into the microscopic master equation, followed by a systematic expansion in $\Omega$. At leading order, we find a set of mean-field equations of motion for the local densities which reads, after taking a naive continuum limit:

$$
\partial_{t} \rho^{ \pm}=-\nabla \Gamma\left\{\left[\rho^{ \pm} \overleftrightarrow{\nabla}\left(1-\rho^{+}-\rho^{-}\right) \pm \varepsilon \hat{\mathbf{x}}_{\|} \rho^{ \pm}\left(1-\rho^{+}-\rho^{-}\right)\right]+\gamma\left[\rho^{ \pm} \overleftrightarrow{\nabla} \rho^{\mp} \pm \varepsilon \hat{\mathbf{x}}_{\|} \rho^{ \pm} \rho^{\mp}\right]\right\}
$$

where

$$
\Gamma=\left(\begin{array}{cc}
\Gamma_{\perp} & 0 \\
0 & \Gamma_{\|}
\end{array}\right)
$$

is the diffusion-matrix. $\quad \boldsymbol{\Gamma}_{\perp}$ is diagonal and isotropic in the $(d-1)$-dimensional subspace, thus characterized by a number $\Gamma_{\perp} . \quad \stackrel{\leftrightarrow}{\nabla}$ is the asymmetric gradient operator, acting on any two functions $f$ and $g$ according to $f \stackrel{\leftrightarrow}{\nabla} g=f \nabla g-g \nabla f . \quad \varepsilon$ is the coarse-grained bias and $\hat{\mathbf{x}}_{\|}$is the unit vector along the $x_{\|}$direction. Note that at the mean-field level we also obtain explicit expressions for the diffusion matrix and the bias 23]: $\Gamma_{\perp}=1$, $\Gamma_{\|}=\left(1+e^{-|E|}\right) / 2$ and $\varepsilon=2 \tanh (E / 2)$. Of course, these may be modified by renormalization.

The continuity equation (9) admits both homogeneous and inhomogeneous $t$-independent solutions, associated with the disordered and the blocked phases. The former is our focus here. To ease comparison with simulation data, we choose equal densities for both species: $\rho^{ \pm}(\mathbf{x}, t)=\bar{\rho}$. This solution describes the steady state at the mean-field level.

At the next order in the $\Omega$-expansion, we find a Fokker-Planck equation for the fluctuating part, $\chi^{ \pm}$. For our purposes, the equivalent Langevin equation is more transparent. At this order, its deterministic part is linear and the (conserved) noise is Gaussian. After defining the "reduced" average density $\tilde{\rho} \equiv(1-\gamma) \bar{\rho}$ and $\delta \equiv(3-\gamma) /(1-\gamma)$, and focusing on the fluctuations about the homogeneous phase, the result is:

$$
\partial_{t} \chi^{\alpha}(\mathbf{x}, t)=\mathcal{L}^{\alpha \beta}(\boldsymbol{\nabla}) \chi^{\beta}(\mathbf{x}, t)-\nabla \boldsymbol{\eta}^{\alpha}(\mathbf{x}, t)
$$

where the drift matrix is given by

$$
\left(\mathcal{L}^{\alpha \beta}(\nabla)\right)=\left(\begin{array}{cc}
(1-\tilde{\rho}) \boldsymbol{\nabla} \Gamma \boldsymbol{\nabla}-(1-\delta \tilde{\rho}) \varepsilon \Gamma_{\|} \partial_{\|} & \tilde{\rho} \nabla \Gamma \nabla+\tilde{\rho} \varepsilon \Gamma_{\|} \partial_{\|} \\
\tilde{\rho} \nabla \Gamma \nabla-\tilde{\rho} \varepsilon \Gamma_{\|} \partial_{\|} & (1-\tilde{\rho}) \nabla \Gamma \nabla+(1-\delta \tilde{\rho}) \varepsilon \Gamma_{\|} \partial_{\|}
\end{array}\right)
$$

and summation over repeated indices is implied in (11) and in the following. The $\boldsymbol{\eta}^{ \pm}(\mathbf{x}, t)$ are Gaussian white noise terms, with average and second moment:

$$
\begin{aligned}
\left\langle\eta_{i}^{\alpha}(\mathbf{x}, t)\right\rangle & =0 \\
\left\langle\eta_{i}^{\alpha}(\mathbf{x}, t) \eta_{j}^{\beta}\left(\mathbf{x}^{\prime}, t^{\prime}\right)\right\rangle & =2 \sigma_{i j}^{\alpha \beta} \delta\left(\mathbf{x}-\mathbf{x}^{\prime}\right) \delta\left(t-t^{\prime}\right),
\end{aligned}
$$

where $\alpha, \beta=+,-; i, j=1,2, \ldots d$. Due to the bias, the noise matrices $\left(\sigma_{i j}^{\alpha \beta}\right)=\sigma^{\alpha \beta}$ are diagonal but not proportional to the unit matrix:

$$
\boldsymbol{\sigma}^{\alpha \beta}=\left(\begin{array}{cc}
\boldsymbol{\sigma}_{\perp}^{\alpha \beta} & \mathbf{0} \\
\mathbf{0} & \sigma_{\|}^{\alpha \beta}
\end{array}\right)
$$


Note that $\boldsymbol{\sigma}^{\alpha \beta}$ is symmetric and due to charge symmetry, we also have $\boldsymbol{\sigma}^{++}=\boldsymbol{\sigma}^{--}$. Similar to $\boldsymbol{\Gamma}_{\perp}, \boldsymbol{\sigma}_{\perp}^{\alpha \beta}$ is diagonal and isotropic in the $(d-1)$-dimensional subspace, characterized by a number $\sigma_{\perp}^{\alpha \beta}$. In the absence of the drive, our model reduces to an equilibrium system, so that the fluctuation dissipation theorem (FDT) holds. In our case, this guarantees $\boldsymbol{\sigma}^{\alpha \beta} \propto \boldsymbol{\Gamma}$, or, more specifically,

$$
\begin{aligned}
& \boldsymbol{\sigma}^{++}=\left[\bar{\rho}(1-2 \bar{\rho})+\gamma \bar{\rho}^{2}\right] \boldsymbol{\Gamma} \\
& \boldsymbol{\sigma}^{+-}=\left[-\gamma \bar{\rho}^{2}\right] \boldsymbol{\Gamma}
\end{aligned}
$$

[23]. However, when driven, this proportionality does not hold in generic ranges of $\gamma$ and $\bar{\rho}$, in that the diffusion and noise matrices are renormalized differently by the drive $\varepsilon$, similar to the situation in the driven single species case 24. Finally, we point out that there is a correlation between $\boldsymbol{\eta}^{+}$and $\boldsymbol{\eta}^{-}$due to the fact that charge exchange is allowed. This effect is captured by the matrix $\boldsymbol{\sigma}^{+-}$which is expected to be proportional to $\gamma$ and negative definite for non-zero drive as well.

\section{A. Steady-state structure factors}

Eqns. (11, 14 are linear equations which are easily solved in Fourier space. Introducing the Fourier components for the fluctuations

$$
\chi^{ \pm}(\mathbf{k}, \omega)=\int d t d^{d} x \chi^{ \pm}(\mathbf{x}, t) e^{-i(\omega t+\mathbf{k x})}
$$

and similar ones for the noise, so that

$$
\begin{aligned}
\left\langle\eta_{i}^{\alpha}(\mathbf{k}, \omega)\right\rangle & =0 \\
\left\langle\eta_{i}^{\alpha}(\mathbf{k}, \omega) \eta_{j}^{\beta}\left(\mathbf{k}^{\prime}, \omega^{\prime}\right)\right\rangle & =2 \sigma_{i j}^{\alpha \beta}\left[(2 \pi)^{d+1} \delta\left(\mathbf{k}+\mathbf{k}^{\prime}\right) \delta\left(\omega+\omega^{\prime}\right)\right]
\end{aligned}
$$

the solution to (11) is simply:

$$
\chi^{\alpha}(\mathbf{k}, \omega)=\left(L^{-1}\right)^{\alpha \beta} i \mathbf{k} \boldsymbol{\eta}^{\beta}(\mathbf{k}, \omega)
$$

where

$$
L^{\alpha \beta}(\mathbf{k}, \omega) \equiv \mathcal{L}^{\alpha \beta}(i \mathbf{k})-i \omega \delta^{\alpha \beta} .
$$

Note that, in $\mathbf{k}$ space, $\left(\mathcal{L}^{++}, \mathcal{L}^{--}\right)$and $\left(\mathcal{L}^{+-}, \mathcal{L}^{-+}\right)$are complex conjugate pairs.

Not surprisingly, $\left\langle\chi^{ \pm}(\mathbf{k}, \omega)\right\rangle=0$, consistent with the decomposition (8). The two-point correlations of $\chi^{ \pm}(\mathbf{k}, \omega)$ are just the dynamic structure factors, defined as

$$
S^{\alpha \beta}(\mathbf{k}, \omega)\left[(2 \pi)^{d+1} \delta\left(\mathbf{k}+\mathbf{k}^{\prime}\right) \delta\left(\omega+\omega^{\prime}\right)\right] \equiv\left\langle\chi^{\alpha}(\mathbf{k}, \omega) \chi^{\beta}\left(\mathbf{k}^{\prime}, \omega^{\prime}\right)\right\rangle .
$$

Using (18) and (17), the two independent $S$ 's follow:

$$
\begin{aligned}
& S^{++}(\mathbf{k}, \omega)=\frac{2 \mathbf{k} \boldsymbol{\sigma}^{++} \mathbf{k}}{|\operatorname{det}(L)|^{2}}\left(\left|L^{--}\right|^{2}+\left|L^{+-}\right|^{2}\right)-\frac{2 \mathbf{k} \boldsymbol{\sigma}^{+-} \mathbf{k}}{|\operatorname{det}(L)|^{2}} 2 \operatorname{Re}\left\{L^{--} L^{-+}\right\} \\
& S^{+-}(\mathbf{k}, \omega)=-\frac{2 \mathbf{k} \boldsymbol{\sigma}^{++} \mathbf{k}}{|\operatorname{det}(L)|^{2}} L^{+-}\left(\left(L^{++}\right)^{*}+L^{--}\right)+\frac{2 \mathbf{k} \boldsymbol{\sigma}^{+-} \mathbf{k}}{|\operatorname{det}(L)|^{2}}\left(\left(L^{++}\right)^{*} L^{--}+\left(L^{+-}\right)^{2}\right) .
\end{aligned}
$$

To compare directly with simulations, we need the steady-state structure factors

$$
S^{\alpha \beta}(\mathbf{k})\left[(2 \pi)^{d} \delta\left(\mathbf{k}+\mathbf{k}^{\prime}\right)\right] \equiv\left\langle\chi^{\alpha}(\mathbf{k}, t) \chi^{\beta}\left(\mathbf{k}^{\prime}, t\right)\right\rangle,
$$

which are easily obtained from (21) by an integration over $\omega$, using the residue theorem and noting that the two zeros of $\operatorname{det}(L)$ simply correspond to the two stable eigenvalues of $\mathcal{L}$ :

$$
\omega_{1,2}=-i \frac{\operatorname{Tr}(\mathcal{L})}{2} \pm \sqrt{\operatorname{det}(\mathcal{L})-\left(\frac{\operatorname{Tr}(\mathcal{L})}{2}\right)^{2}}
$$


To ensure that the system is within the linear stability region of the disordered phase, we must have $\operatorname{Im} \omega_{1,2}>0$. Since $-\frac{1}{2} \operatorname{Tr}(\mathcal{L})=(1-\tilde{\rho}) \mathbf{k} \boldsymbol{\Gamma} \mathbf{k}$ is automatically positive definite, we only require $\operatorname{det}(\mathcal{L})>0$ for all $\mathbf{k} \neq 0$. Collecting, we find:

$$
\begin{aligned}
& S^{++}(\mathbf{k})=\frac{\mathbf{k} \boldsymbol{\sigma}^{++} \mathbf{k}}{-\frac{1}{2} \operatorname{Tr}(\mathcal{L})} \frac{\left|\mathcal{L}^{--}\right|^{2}}{\operatorname{det}(\mathcal{L})}-\frac{\mathbf{k} \boldsymbol{\sigma}^{+-} \mathbf{k}}{-\frac{1}{2} \operatorname{Tr}(\mathcal{L})} \frac{\operatorname{Re}\left\{\mathcal{L}^{--} \mathcal{L}^{-+}\right\}}{\operatorname{det}(\mathcal{L})} \\
& S^{+-}(\mathbf{k})=-\frac{\mathbf{k} \boldsymbol{\sigma}^{++} \mathbf{k}}{-\frac{1}{2} \operatorname{Tr}(\mathcal{L})} \frac{\mathcal{L}^{--} \mathcal{L}^{+-}}{\operatorname{det}(\mathcal{L})}+\frac{\mathbf{k} \boldsymbol{\sigma}^{+-} \mathbf{k}}{-\frac{1}{2} \operatorname{Tr}(\mathcal{L})} \frac{\mathcal{L}^{--} \operatorname{Re}\left\{\mathcal{L}^{--}\right\}+i \mathcal{L}^{+-} \operatorname{Im}\left\{\mathcal{L}^{+-}\right\}}{\operatorname{det}(\mathcal{L})}
\end{aligned}
$$

so that, with the help of (12), we finally obtain:

$$
\begin{aligned}
S^{++}(\mathbf{k}) & =\frac{(1-\tilde{\rho})}{(1-2 \tilde{\rho})} \frac{\mathbf{k} \boldsymbol{\sigma}^{++} \mathbf{k}}{\mathbf{k} \boldsymbol{\Gamma} \mathbf{k}} \frac{(\mathbf{k} \boldsymbol{\Gamma} \mathbf{k})^{2}+\frac{(1-\delta \tilde{\tilde{s}})^{2}}{(1-\tilde{\rho})^{2}} \varepsilon^{2} \Gamma_{\|}^{2} k_{\|}^{2}}{(\mathbf{k} \boldsymbol{\Gamma})^{2}+4 m^{2} \Gamma_{\|} k_{\|}^{2}} \\
& -\frac{\tilde{\rho}}{(1-2 \tilde{\rho})} \frac{\mathbf{k} \boldsymbol{\sigma}^{+-} \mathbf{k}}{\mathbf{k} \boldsymbol{\mathbf { k }}} \frac{(\mathbf{k} \boldsymbol{\Gamma} \mathbf{k})^{2}+\frac{(1-\delta \tilde{\rho})}{(1-\tilde{\rho})} \varepsilon^{2} \Gamma_{\|}^{2} k_{\|}^{2}}{(\mathbf{k} \boldsymbol{\Gamma} \mathbf{k})^{2}+4 m^{2} \Gamma_{\|} k_{\|}^{2}} \\
\operatorname{Re}\left\{S^{+-}(\mathbf{k})\right\} & =-\frac{\tilde{\rho}}{(1-2 \tilde{\rho})} \frac{\mathbf{k} \boldsymbol{\sigma}^{++} \mathbf{k}}{\mathbf{k} \boldsymbol{\Gamma} \mathbf{k}} \frac{(\mathbf{k} \boldsymbol{\Gamma} \mathbf{k})^{2}-\frac{(1-\delta \tilde{\rho})}{(1-\tilde{\rho})} \varepsilon^{2} \Gamma_{\|}^{2} k_{\|}^{2}}{(\mathbf{k} \boldsymbol{\Gamma} \mathbf{k})^{2}+4 m^{2} \Gamma_{\|} k_{\|}^{2}} \\
& +\frac{(1-\tilde{\rho})}{(1-2 \tilde{\rho})} \frac{\mathbf{k} \boldsymbol{\sigma}^{+-} \mathbf{k}}{\mathbf{k} \boldsymbol{\mathbf { k }}} \frac{(\mathbf{k} \boldsymbol{\Gamma} \mathbf{k})^{2}-\frac{\tilde{\rho}^{2}}{(1-\tilde{\rho})^{2}} \varepsilon^{2} \Gamma_{\|}^{2} k_{\|}^{2}}{(\mathbf{k} \boldsymbol{\Gamma} \mathbf{k})^{2}+4 m^{2} \Gamma_{\|} k_{\|}^{2}} \\
\operatorname{Im}\left\{S^{+-}(\mathbf{k})\right\}= & \frac{\tilde{\rho}(2-(1+\delta) \tilde{\rho})}{(1-\tilde{\rho})(1-2 \tilde{\rho})} \frac{\left(\mathbf{k} \boldsymbol{\sigma}^{++} \mathbf{k}\right) \varepsilon \Gamma_{\|} k_{\|}}{(\mathbf{k} \mathbf{k})^{2}+4 m^{2} \Gamma_{\|} k_{\|}^{2}} \\
& -\frac{(1-\tilde{\rho})(1-\delta \tilde{\rho})+\tilde{\rho}^{2}}{(1-\tilde{\rho})(1-2 \tilde{\rho})} \frac{\left(\mathbf{k} \boldsymbol{\sigma}^{+-} \mathbf{k}\right) \varepsilon \Gamma_{\|} k_{\|}}{(\mathbf{k} \boldsymbol{\Gamma} \mathbf{k})^{2}+4 m^{2} \Gamma_{\|} k_{\|}^{2}},
\end{aligned}
$$

To simplify the notation, we have defined a "mass" (in the field theory sense), $m$, via

$$
4 m^{2} \equiv \frac{(1-\delta \tilde{\rho})^{2}-\tilde{\rho}^{2}}{1-2 \tilde{\rho}} \varepsilon^{2} \Gamma_{\|}=\frac{(1-2 \bar{\rho})(1-(2-\gamma) 2 \bar{\rho})}{(1-(1-\gamma) 2 \bar{\rho})} \varepsilon^{2} \Gamma_{\|}
$$

Its role is to mark the linear stability boundary, which, in the limit $\varepsilon L \rightarrow \infty$, is given precisely by $m^{2}=0$. Otherwise, for finite $\varepsilon L$, the system does not reach the stability limit as long as $(\varepsilon L / 2 \pi)^{2}<(1-(1-\gamma) 2 \bar{\rho}) /(1-2 \bar{\rho})((2-\gamma) 2 \bar{\rho}-1)$ is satisfied [16]. Thus, it is sufficient to impose $m^{2}>0$, i.e., $\bar{\rho}<\frac{1}{2(2-\gamma)}$, to keep the system in the homogeneous phase.

Similar to the driven lattice gas [2,3] and the two-species model studied earlier [20], these structure factors are all singular at the origin. The singularity takes the form of a discontinuity, either in the function itself or one of its derivatives. In particular, both $S^{++}$and $\operatorname{Re}\left\{S^{+-}\right\}$are discontinuous, so that the ratios

$$
\frac{\lim _{k_{\| \rightarrow 0} \rightarrow} S^{++}\left(\mathbf{0}, k_{\|}\right)}{\lim _{\mathbf{k}_{\perp} \rightarrow \mathbf{0}} S^{++}\left(\mathbf{k}_{\perp}, 0\right)}=\frac{1-2 \tilde{\rho}}{(1-\delta \tilde{\rho})^{2}-\tilde{\rho}^{2}} \frac{(1-\delta \tilde{\rho})^{2}}{(1-\tilde{\rho})^{2}} \frac{\frac{\sigma_{\|}^{++}}{\Gamma_{\|}}-\frac{\tilde{\rho}}{1-\delta \tilde{\rho}} \frac{\sigma_{\|}^{+-}}{\Gamma_{\|}}}{\frac{\sigma_{\perp}^{++}}{\Gamma_{\perp}}-\frac{\tilde{\rho}}{1-\tilde{\rho}} \frac{\sigma_{\perp}^{+-}}{\Gamma_{\perp}}}
$$

and

$$
\frac{\lim _{k_{\|} \rightarrow 0} \operatorname{Re}\left\{S^{+-}\left(\mathbf{0}, k_{\|}\right)\right\}}{\lim _{\mathbf{k}_{\perp} \rightarrow \mathbf{0}} \operatorname{Re}\left\{S^{+-}\left(\mathbf{k}_{\perp}, 0\right)\right\}}=-\frac{1-2 \tilde{\rho}}{(1-\delta \tilde{\rho})^{2}-\tilde{\rho}^{2}} \frac{1-\delta \tilde{\rho}}{1-\tilde{\rho}} \frac{\frac{\sigma_{\|}^{++}}{\Gamma_{\|}}-\frac{\tilde{\rho}}{1-\delta \tilde{\rho}} \frac{\sigma_{\|}^{+-}}{\Gamma_{\|}}}{\frac{\sigma_{\perp}^{++}}{\Gamma_{\perp}}-\frac{1-\tilde{\rho}}{\tilde{\rho}} \frac{\sigma_{\perp}^{+-}}{\Gamma_{\perp}}} .
$$

are in general different from unity. In contrast, $\operatorname{Im}\left\{S^{+-}(\mathbf{k})\right\}$ vanishes for $\mathbf{k} \rightarrow \mathbf{0}$ in any direction. Here, discontinuities occur in higher derivatives. Unlike in the driven Ising lattice gas, these singularities do not simply originate in the generic FDT-breaking relation $\frac{\sigma_{\|}^{\alpha \beta}}{\Gamma_{\|}} \neq \frac{\sigma_{\perp}^{\alpha \beta}}{\Gamma_{\perp}}$, but also in the specifics of this particular driven system, reflected in the first factor on the right hand side of (27,28). It is a monotonically increasing function of $\bar{\rho}$, reaching $\infty$ at $\bar{\rho}=\frac{1}{2(2-\gamma)}$. As a result, the amplitudes of the discontinuities diverge as the system approaches the stability limit of the homogeneous phase. 


\section{B. Equal-time spatial correlations}

The equal-time correlation functions $G^{\alpha \beta}(\mathbf{x}) \equiv\left\langle\chi^{\alpha}\left(\mathbf{x}^{\prime}+\mathbf{x}, t\right) \chi^{\beta}\left(\mathbf{x}^{\prime}, t\right)\right\rangle$ are just the Fourier transforms of the structure factors,

$$
G^{\alpha \beta}(\mathbf{x})=\int \frac{d^{d} k}{(2 \pi)^{d}} S^{\alpha \beta}(\mathbf{k}) e^{i \mathbf{k x}}
$$

independent of $\mathbf{x}^{\prime}$ by virtue of translational invariance. To simplify the transforms, we introduce some changes in notation. First, we rescale the lengths and momenta $\mathbf{x}_{\perp} \rightarrow \mathbf{x}_{\perp} / \Gamma_{\perp}^{\frac{1}{2}}, x_{\|} \rightarrow x_{\|} / \Gamma_{\|}^{\frac{1}{2}} ; \mathbf{k}_{\perp} \rightarrow \Gamma_{\perp}^{\frac{1}{2}} \mathbf{k}_{\perp}, k_{\|} \rightarrow \Gamma_{\|}^{\frac{1}{2}} k_{\|}$so that $\boldsymbol{\Gamma}$ becomes the unit matrix. Further, we let $\sigma_{\perp}^{\alpha \beta} \rightarrow \sigma_{\perp}^{\alpha \beta} / \Gamma_{\perp}, \sigma_{\|}^{\alpha \beta} \rightarrow \sigma_{\|}^{\alpha \beta} / \Gamma_{\|}$. After some algebra, we can recast the structure factors in much more compact form:

$$
\begin{aligned}
S^{++}(\mathbf{k}) & =\frac{\mathbf{k} \boldsymbol{\sigma}^{1} \mathbf{k}}{k^{2}}-\left(\mathbf{k} \boldsymbol{\sigma}^{2} \mathbf{k}\right) \frac{k^{2}}{k^{4}+4 m^{2} k_{\|}^{2}} \\
\operatorname{Re}\left\{S^{+-}(\mathbf{k})\right\} & =\frac{\mathbf{k} \boldsymbol{\sigma}^{3} \mathbf{k}}{k^{2}}-\left(\mathbf{k} \boldsymbol{\sigma}^{4} \mathbf{k}\right) \frac{k^{2}}{k^{4}+4 m^{2} k_{\|}^{2}} \\
\operatorname{Im}\left\{S^{+-}(\mathbf{k})\right\} & =\left(\mathbf{k} \boldsymbol{\sigma}^{5} \mathbf{k}\right) \frac{\varepsilon \Gamma_{\|}^{\frac{1}{2}} k_{\|}}{k^{4}+4 m^{2} k_{\|}^{2}},
\end{aligned}
$$

where $k=|\mathbf{k}|$ and

$$
\begin{aligned}
\boldsymbol{\sigma}^{1} & =\frac{(1-\delta \tilde{\rho})^{2}}{(1-\tilde{\rho})\left((1-\delta \tilde{\rho})^{2}-\tilde{\rho}^{2}\right)} \boldsymbol{\sigma}^{++}-\frac{(1-\tilde{\rho}) \tilde{\rho}}{(1-\tilde{\rho})\left((1-\delta \tilde{\rho})^{2}-\tilde{\rho}^{2}\right)} \boldsymbol{\sigma}^{+-} \\
\boldsymbol{\sigma}^{2} & =\left[\frac{(1-\delta \tilde{\rho})^{2}(1-2 \tilde{\rho})}{(1-\tilde{\rho})^{2}\left((1-\delta \tilde{\rho})^{2}-\tilde{\rho}^{2}\right)}-1\right] \frac{(1-\tilde{\rho})}{(1-2 \tilde{\rho})} \boldsymbol{\sigma}^{++} \\
& -\left[\frac{(1-\delta \tilde{\rho})(1-2 \tilde{\rho})}{(1-\tilde{\rho})\left((1-\delta \tilde{\rho})^{2}-\tilde{\rho}^{2}\right)}-1\right] \frac{\tilde{\rho}}{(1-2 \tilde{\rho})} \boldsymbol{\sigma}^{+-} \\
\boldsymbol{\sigma}^{3} & =\frac{(1-\tilde{\rho}) \tilde{\rho}}{(1-\tilde{\rho})\left((1-\delta \tilde{\rho})^{2}-\tilde{\rho}^{2}\right)} \boldsymbol{\sigma}^{++}-\frac{\tilde{\rho}^{2}}{(1-\tilde{\rho})\left((1-\delta \tilde{\rho})^{2}-\tilde{\rho}^{2}\right)} \boldsymbol{\sigma}^{+-} \\
\boldsymbol{\sigma}^{4} & =\left[\frac{(1-\delta \tilde{\rho})(1-2 \tilde{\rho})}{(1-\tilde{\rho})\left((1-\delta \tilde{\rho})^{2}-\tilde{\rho}^{2}\right)}+1\right] \frac{\tilde{\rho}}{(1-2 \tilde{\rho})} \boldsymbol{\sigma}^{++} \\
& -\left[\frac{\tilde{\rho}(1-2 \tilde{\rho})}{(1-\tilde{\rho})^{2}\left((1-\delta \tilde{\rho})^{2}-\tilde{\rho}^{2}\right)}+1\right] \frac{(1-\tilde{\rho})}{(1-2 \tilde{\rho})} \boldsymbol{\sigma}^{+-} \\
\boldsymbol{\sigma}^{5} & =\frac{\tilde{\rho}(2-(1+\delta) \tilde{\rho})}{(1-\tilde{\rho})(1-2 \tilde{\rho})} \boldsymbol{\sigma}^{++}-\frac{(1-\tilde{\rho})(1-\delta \tilde{\rho})+\tilde{\rho}^{2}}{(1-\tilde{\rho})(1-2 \tilde{\rho})} \boldsymbol{\sigma}^{+-} .
\end{aligned}
$$

For the following, only two major properties of the $\boldsymbol{\sigma}$ 's, rather than their detailed expressions, are important. First, they are all positive definite within the stability limit of the homogeneous phase, and second, they are diagonal but generically not proportional to the unit matrix, since they are related to the noise matrices. Now, referring the details of the integrations to Appendix A, the transforms can be carried out exactly. Writing $r \equiv|\mathbf{x}|, r_{\perp} \equiv\left|\mathbf{x}_{\perp}\right|$ and $r_{\|} \equiv\left|x_{\|}\right|$, we define

$$
\begin{aligned}
E(\mathbf{x}) & \equiv \frac{\Gamma\left(\frac{d}{2}-1\right)}{4 \pi^{\frac{d}{2}}} \frac{1}{r^{d-2}} \\
F_{1}(\mathbf{x}) & \equiv \frac{\cosh \left(m x_{\|}\right)}{(2 \pi)^{\frac{d}{2}}}\left(\frac{m}{r}\right)^{\frac{d-2}{2}} K_{\frac{d-2}{2}}(m r) \\
F_{2}(\mathbf{x}) & \equiv \frac{\sinh \left(m x_{\|}\right)}{(2 \pi)^{\frac{d}{2}}}\left(\frac{m}{r}\right)^{\frac{d-2}{2}} K_{\frac{d-2}{2}}(m r),
\end{aligned}
$$

where $\Gamma(z)$ is the Gamma function and $K_{\nu}(z)$ is the modified Bessel function. The correlations can then be expressed in terms of these three functions: 


$$
\begin{aligned}
G^{++}(\mathbf{x}) & =-\nabla \sigma^{1} \nabla E(\mathbf{x})+\nabla \sigma^{2} \nabla F_{1}(\mathbf{x}) \\
G_{e}^{+-}(\mathbf{x})= & -\nabla \sigma^{3} \nabla E(\mathbf{x})+\nabla \sigma^{4} \nabla F_{1}(\mathbf{x}) \\
G_{o}^{+-}(\mathbf{x})= & \frac{\varepsilon \Gamma_{\|}^{\frac{1}{2}}}{2 m} \nabla \sigma^{5} \nabla F_{2}(\mathbf{x}),
\end{aligned}
$$

where $G_{e, o}^{+-}$are the parts of $G^{+-}$even or odd in $x_{\|}$, corresponding to the transforms of the real and imaginary parts of $S^{+-}$. The full correlation is, of course, $G^{+-}(\mathbf{x})=G_{e}^{+-}(\mathbf{x})+G_{o}^{+-}(\mathbf{x})$, reflecting the symmetry (7) of the system in the presence of the field.

Leaving the detailed asymptotic expansions of (33-35) to Appendix B, we only indicate the main features here. Clearly, the discontinuity singularities of the structure factors translate into power-law decays of the correlation functions. In particular, the first terms in (33) and (34) exhibit the well-known $r^{-d}$-decay [25] which is associated with the breaking of the FDT in the presence of conservation laws 5 . In contrast, a discontinuity singularity in higher derivatives is not sufficient to produce the $r^{-d}$ power, as illustrated by the form of $G_{o}^{+-}$, (35)). The second terms in (33) and (34) and the only one in (35) are more subtle, since the exponentials of the hyperbolic and Bessel functions compete. Summarizing our results as $m r \rightarrow \infty$, we find for $r_{\perp} \neq 0$ :

$$
\begin{aligned}
G^{++}(\mathbf{x}) \propto \frac{\sigma_{\|}^{1}-\sigma_{\perp}^{1}}{r^{d}}\left[\frac{r_{\perp}^{2}-(d-1) r_{\|}^{2}}{r^{2}}\right]+\ldots \\
G^{+-}(\mathbf{x}) \simeq G_{e}^{+-}(\mathbf{x}) \propto \frac{\sigma_{\|}^{3}-\sigma_{\perp}^{3}}{r^{d}}\left[\frac{r_{\perp}^{2}-(d-1) r_{\|}^{2}}{r^{2}}\right]+\ldots, \\
G_{o}^{+-}(\mathbf{x}) \propto \ldots
\end{aligned}
$$

where the ... represent exponentially decaying parts. Here, all three key components of the characteristic nonequilibrium power law decays are displayed, namely, the necessity of FDT violation, $\sigma_{\|} / \Gamma_{\|} \neq \sigma_{\perp} / \Gamma_{\perp}$, the dipole amplitude associated with strong anisotropy in the presence of a conservation law, and the $r^{-d}$ itself. We emphasize again that the odd part of the cross-correlations, $G_{o}^{+-}(\mathbf{x})$, is purely short-ranged.

Along the field direction, the behavior of the correlations is more complex. Here, a novel power, $r_{\|}^{-(d+1) / 2}$, emerges, which will dominate over the "FDT-violating" $r^{-d}$, for all $d>1$. Thus, for $r_{\perp}=0$ we have:

$$
\begin{aligned}
G^{++}\left(\mathbf{0}, x_{\|}\right), G_{e}^{+-}\left(\mathbf{0}, x_{\|}\right) & \propto-r_{\|}^{-\frac{d+1}{2}}+\mathcal{O}\left(\max \left\{r_{\|}^{-d}, r_{\|}^{-\frac{d+3}{2}}\right\}\right) \\
G_{o}^{+-}\left(\mathbf{0}, x_{\|}\right) & \propto-\operatorname{sgn}\left(\varepsilon x_{\|}\right) r_{\|}^{-\frac{d+1}{2}}+\mathcal{O}\left(r_{\|}^{-\frac{d+3}{2}}\right) .
\end{aligned}
$$

In the parentheses, we have indicated the next-leading term in the asymptotic expansion of $K_{\frac{d-2}{2}}$. Surprisingly, in $d>3$ even this power is still more relevant than the more familiar $r^{-d}$. We also emphasize that in (37), all proportionality constants are positive. Thus, the explicit factors of $(-1)$ carry information about the structure of particle clusters in this model. In particular, the sign of $G_{o}^{+-}\left(\mathbf{0}, x_{\|}\right)$shows that negative charges prefer to be located 'downfield', rather than 'upfield', from positive ones, as a precursor of the blocking transition. In conclusion, the spatial correlations are dominated by the expected $r^{-d}$ power law, except along the field, where a novel $r_{\|}^{-(d+1) / 2}$ decay takes over. Similar behavior is found if charge exchange is not allowed $(\gamma=0)$ [19]. Thus, this new power law appears to be a generic feature of driven two-species models, associated with the excluded volume constraint and the opposite bias. We should note, however, that it can only be generated in the presence of at least one transverse dimension, i.e., in $d>1$.

\section{The $\gamma=1$ case}

All expressions simplify considerably when we set $\gamma=1$, yet they still capture the essence of this two species model, namely, the non-trivial correlations between opposite charges:

$$
S^{++}(\mathbf{k})=\frac{\mathbf{k} \boldsymbol{\sigma}^{++} \mathbf{k}}{k^{2}}
$$




$$
\begin{aligned}
& \operatorname{Re}\left\{S^{+-}(\mathbf{k})\right\}=\left(\mathbf{k} \boldsymbol{\sigma}^{+-} \mathbf{k}\right) \frac{k^{2}}{k^{4}+4 m^{2} k_{\|}^{2}} \\
& \operatorname{Im}\left\{S^{+-}(\mathbf{k})\right\}=\left(\mathbf{k} \boldsymbol{\sigma}^{+-} \mathbf{k}\right) \frac{-\operatorname{sgn}(\varepsilon) 2 m k_{\|}}{k^{4}+4 m^{2} k_{\|}^{2}},
\end{aligned}
$$

where now $4 m^{2}=(1-2 \bar{\rho})^{2} \varepsilon^{2} \Gamma_{\|}$. No instabilities can occur here: even for $\bar{\rho}=1 / 2$ where the "mass" $m^{2}$ vanishes, homogeneous configurations prevail since the model reduces to a driven one-species model. For generic densities, we note that the form of the ++ structure factor is the same as in the one-species model, due to the fact that + 's cannot distinguish between -'s and holes at the microscopic level. The key question is, of course, whether the $\boldsymbol{\sigma}$ 's (especially $\boldsymbol{\sigma}^{++}$) are proportional to the unit matrix or not. Unfortunately, in the absence of a renormalization group analysis we have to rely on simulations to answer these questions. Based on the results of the previous subsection, it is clear that only $S^{++}(\mathbf{k})$ could possibly produce the $r^{-d}$ power law. However, our simulation results indicate that the internal symmetry of the system, at this particular value of $\gamma$, restores FDT for either species, i.e., the first equation in (15). This is entirely consistent with the fact that the microscopic steady state distribution of either species is uniform, as mentioned in Section II. Thus, correlations will be short ranged, given by a $\delta$-function for identical species and exponential decays for opposite charges, except in the field direction, between opposite species, where:

$$
G^{+-}\left(\mathbf{0}, x_{\|}\right) \simeq 2 \Theta\left(\varepsilon x_{\|}\right) \sigma_{\perp}^{+-} \frac{\sqrt{\frac{\pi}{2}}}{(2 \pi)^{\frac{d}{2}}}\left\{\frac{d-1}{2 m}\left(\frac{m}{r_{\|}}\right)^{\frac{d+1}{2}}+\mathcal{O}\left(\frac{1}{r_{\|}^{\frac{d+3}{2}}}\right)\right\} .
$$

Here $\Theta(x)$ is the step function and $\sigma_{\perp}^{+-}$is always negative. Thus, the novel $r_{\|}^{-(d+1) / 2}$ power law, a key feature of this two-species model, survives in the cross correlation, even in this simplified case.

\section{Distribution of structure factors}

So far, we have focused entirely on the averages of density-density operators. In this final section, we will construct the full probability distributions for these fluctuating quantities, i.e., $\frac{\chi^{+}(\mathbf{k}, t) \chi^{+}(-\mathbf{k}, t)}{V}, \frac{\operatorname{Re}\left[\chi^{+}(\mathbf{k}, t) \chi^{-}(-\mathbf{k}, t)\right]}{V}$ and $\frac{\operatorname{Im}\left[\chi^{+}(\mathbf{k}, t) \chi^{-}(-\mathbf{k}, t)\right]}{V}$, following the method of ref. [26]. Representing these operators by $s^{++}, s_{r}^{+-}$and $s_{i}^{+-}$, we seek their marginal distributions, for each $\mathbf{k}$-vector separately:

$$
\begin{aligned}
& P^{++}\left(s^{++} ; \mathbf{k}\right)=\left\langle\delta\left(\frac{\chi^{+}(\mathbf{k}, t) \chi^{+^{*}}(\mathbf{k}, t)}{V}-s^{++}\right)\right\rangle \\
& P_{r}^{+-}\left(s_{r}^{+-} ; \mathbf{k}\right)=\left\langle\delta\left(\frac{\operatorname{Re}\left[\chi^{+}(\mathbf{k}, t) \chi^{-*}(\mathbf{k}, t)\right]}{V}-s_{r}^{+-}\right)\right\rangle \\
& P_{i}^{+-}\left(s_{i}^{+-} ; \mathbf{k}\right)=\left\langle\delta\left(\frac{\operatorname{Im}\left[\chi^{+}(\mathbf{k}, t) \chi^{-*}(\mathbf{k}, t)\right]}{V}-s_{i}^{+-}\right)\right\rangle .
\end{aligned}
$$

Here, we have used $\chi^{ \pm}(-\mathbf{k}, t)=\chi^{ \pm^{*}}(\mathbf{k}, t)$, since the densities $\chi^{ \pm}(\mathbf{r}, t)$ are real. Also, we have normalized by $V$ in order to obtain a well-defined thermodynamic limit, noting that $(2 \pi)^{d} \delta(\mathbf{k}=\mathbf{0})=V$. In principle, these distributions can be computed explicitly, by inserting the solution $\chi^{ \pm}(\mathbf{k}, t)$ of the Langevin equation (11) into (40) and averaging over the noise, associated with (13). However, given that these distributions are universal [20|, depending only on the linearity of the Langevin equation and the Gaussian nature of the noise, rather than on the specific forms of diffusion and noise matrices, (12) and (14), a detailed calculation is not necessary. Instead, we can refer to the distributions for a simpler case [20], namely the model without charge exchange, since their forms will be identical to the ones we are seeking here. However, some brief comments are in order, to put the results into perspective. For technical reasons, it is simpler to compute the characteristic functions (i.e., Fourier transforms) of (40) first. Denoting these by $\tilde{P}^{\alpha \beta}(\Omega)$, we find that $\tilde{P}^{++}(\Omega)$ has a single pole in the lower half $\Omega$-plane, so that the inverse transform yields an exponential distribution for the non-negative variable $s^{++}$:

$$
P^{++}\left(s^{++} ; \mathbf{k}\right)=\left\{\begin{array}{cl}
\frac{1}{S^{++}(\mathbf{k})} e^{-s^{++} / S^{++}(\mathbf{k})} & \text { if } s^{++} \geq 0 \\
0 & \text { if } s^{++}<0
\end{array}\right.
$$


Here, $S^{++}(\mathbf{k})$ is just the average structure factor, and we will refer to $1 / S^{++}(\mathbf{k})$ as the "decay factor" of the exponential. In contrast, both $\tilde{P}_{r}^{+-}(\Omega)$ and $\tilde{P}_{i}^{+-}(\Omega)$ exhibit two poles, one $\left(\Omega_{-}\right)$being on the negative, and one $\left(\Omega_{+}\right)$on the positive imaginary axis,

$$
\Omega_{\mp}=\frac{2 i}{\Delta}\left(\operatorname{Re}\left[S^{+-}(\mathbf{k})\right] \mp \sqrt{\Delta+\left(\operatorname{Re}\left[S^{+-}(\mathbf{k})\right]\right)^{2}}\right)
$$

where $\Delta \equiv\left|S^{++}(\mathbf{k})\right|^{2}-\left|S^{+-}(\mathbf{k})\right|^{2}>0$. The inverse transforms also result in exponential distributions, characterized however by two distinct decay factors $\left|\Omega_{+}\right|$and $\left|\Omega_{-}\right|$:

$$
P_{r}^{+-}\left(s_{r}^{+-} ; \mathbf{k}\right)=\left\{\begin{array}{cl}
\frac{1}{N} e^{-\left|\Omega_{-}\right| s_{r}^{+-}} & \text {if } s_{r}^{+-} \geq 0 \\
\frac{1}{N} e^{\left|\Omega_{+}\right| s_{r}^{+-}} & \text {if } s_{r}^{+-}<0
\end{array}\right.
$$

with $N=\sqrt{\Delta+\left(\operatorname{Re}\left[S^{+-}(\mathbf{k})\right]\right)^{2}}$. The distribution of $\frac{\operatorname{Im}\left[\chi^{+}(t) \chi^{-*}(t)\right]}{V}$, i.e., $P_{i}^{+-}$, follows from $P_{r}^{+-}$by just interchanging $\operatorname{Re}\left[S^{+-}(\mathbf{k})\right]$ and $\operatorname{Im}\left[S^{+-}(\mathbf{k})\right]$ in Eqs. (42, 433).

To summarize, all three distributions are asymmetric exponentials, with $P^{++}$representing the most extreme case. Due to this structure, their standard deviations are always greater or equal than the averages, so that fluctuations will never be "small", in the usual sense [20,26].

\section{DISCUSSION}

Finally, let us turn to comparisons with simulation results. Typically, we find that power law tails are much more difficult to observe than in the single-species case [25]. Apparently, their amplitudes are rather small, so that the data are obscured by either critical singularities or finite size effects, depending on the points in the phase diagram which we choose to investigate. Thus, we focus on the structure factors. Using a standard least-square routine, we fitted our analytical results (eqs. (30) and (38) before rescaling) to our simulation data. The fit was done simultaneously for the three $S$ 's using the smallest $5 \times 11$ non-zero $\mathbf{k}$ vectors. The agreement is quite good, especially considering that the theoretical results are based on a linearized Langevin equation, but we note the following: for the $\gamma=0.02$ case (Fig. 1), despite being in the homogeneous phase, the system was relatively close to the continuous transition [16], with $m \sim 4 \times 10^{-2}$ corresponding to a correlation length $\xi \sim 25$ in units of the lattice constant. In particular, "longitudinal" parameters, such as $\Gamma_{\|}$and the $\sigma_{\|}$'s, seem to suffer considerable renormalizations here. On the other hand, the "transverse" parameters, $\Gamma_{\perp}$ and $\sigma_{\perp}$, appear to obey Eqn. (15). In that sense, the FDT is satisfied within the transverse subspace. To illustrate this feature, we combine (15), written for the transverse parameters, with the explicit form of the structure factors (25) for $k_{\|}=0$. This yields the exact "finite size" amplitudes, completely independent of $\mathbf{k}_{\perp}: S^{++}\left(\mathbf{k}_{\perp}, 0\right)=\bar{\rho}(1-\bar{\rho})$ and $S^{+-}\left(\mathbf{k}_{\perp}, 0\right)=-\bar{\rho}^{2}$, in perfect agreement with the simulations. In the full $d$-dimensional space, however, the FDT is of course violated: As a result of the coarse-graining effect in the field direction, we generically found $\sigma_{\|} / \Gamma_{\|} \neq \sigma_{\perp} / \Gamma_{\perp}$ for the rescaled "noise" matrices. In particular we had $\sigma_{\|}^{1} / \Gamma_{\|}=0.833 \sigma_{\perp}^{1} / \Gamma_{\perp}$, predicting the typical FDT-violating power law.

For $\gamma=1.00$ (Fig. 2), $S^{++}(\mathbf{k})$ is completely flat, as we expected, indicating that $\sigma_{\|}^{++} / \Gamma_{\|}=\sigma_{\perp}^{++} / \Gamma_{\perp}$. Moreover, the value of this constant is just $\bar{\rho}(1-\bar{\rho})$, again consistent with (15). In contrast, $S^{+-}(\mathbf{k})$ clearly exhibits the structure of Eqs. (38). Here the system is far from transitions $(\xi \sim 3)$, so that critical fluctuations are completely avoided. Consequently, using $m=\frac{1}{2}(1-2 \bar{\rho})|\varepsilon| \Gamma_{\|}^{\frac{1}{2}}$ with the mean-field parameters produces a "mass" closely matching the one obtained from the fit.

Now, we turn to a comparison of the analytical results for the structure factor distributions with the simulations, summarized in Figs. 3 and 1 , for the two smallest wave vectors, respectively. The control parameters were the same as those of Fig. 1. Again, the agreement between our Gaussian theory and the data is quite impresssive. The ' ++ ' histograms show simple exponential decay [26], while the ' +- ' histograms clearly represent asymmetric exponential distributions. To test the theoretical prediction, namely, that the slopes of the histograms are determined entirely by the structure factor averages, we simply measured the latter, i.e., $S^{++}, \operatorname{Re} S^{+-}$and $\operatorname{Im} S^{+-}$. We then inserted the measured averages into the theoretical relations for the decay factors. Clearly, the ' ++ ' case is particularly simple since the decay factor is just the inverse of $S^{++}$itself. For the two ' +- ' distributions, the decay factors $\left|\Omega_{\mp}\right|$, given by (42), are considerably less trivial, but the agreement is nevertheless remarkable. Here, renormalizations can obviously also occur, but can be absorbed into the effective parameters of the theory, leaving the form of the structure factor distributions invariant. Moreover, they are automatically captured by the measured structure factors, so that they do not spoil the agreement between data and theory here. However, we must avoid critical fluctuations since these fall out of the scope of a linear theory. 
In summary, using both simulations and analytic techniques, we have examined the structure factors in a simple model of biased diffusion of two species. We calculated the corresponding spatial correlations, finding not only the expected power law decay, $r^{-d}$, typical for non-equilibrium steady states of conserved systems in the presence of strong anisotropy, but also a novel power, $r_{\|}^{-(d+1) / 2}$, for correlations along the bias, characteristic for two-species models. We also investigated the full distribution functions for the structure factors, being universal asymmetric distributions. The general agreement between simulations and a Gaussian field theory is surprisingly good, while we await a renormalization group analysis of the continuum theory of the model in order to make more detailed comparisons closer to the continuous transition.

\section{ACKNOWLEDGEMENTS}

We thank R.K.P. Zia, Z. Toroczkai and S. Sandow for many stimulating discussions. This research is supported in part by grants from the National Science Foundation through the Division of Materials Research .

\section{APPENDIX A: MOMENTUM-SPACE INTEGRALS FOR THE CORRELATION FUNCTIONS}

From Eqn. (30) we see that we need three basic type of integrals. Although the first one is well known, we list it for completeness:

$$
E(\mathbf{x}) \equiv \int \frac{d^{d} k}{(2 \pi)^{d}} \frac{e^{i \mathbf{k x}}}{k^{2}}=\frac{\Gamma\left(\frac{d}{2}-1\right)}{4 \pi^{\frac{d}{2}}} \frac{1}{r^{d-2}},
$$

Then, if $\boldsymbol{\sigma}$ is diagonal and isotropic in the $d-1$ dimensional transverse subspace but not a multiple of the unit matrix, it is easy to compute

$$
\nabla \sigma \nabla E(\mathbf{x})=-\sigma_{\perp} \delta(\mathbf{x})-\left(\sigma_{\|}-\sigma_{\perp}\right) \frac{\Gamma\left(\frac{d}{2}\right)}{2 \pi^{\frac{d}{2}}} \frac{r_{\perp}^{2}-(d-1) r_{\|}^{2}}{r^{d+2}}
$$

Next, we will outline a formal way to obtain the other two required momentum integrals. For a more rigorous treatment see [23]. We define $F_{1}$ and $F_{2}$ as follows:

$$
\begin{aligned}
& F_{1}(\mathbf{x}) \equiv \int \frac{d^{d} k}{(2 \pi)^{d}} \frac{e^{i \mathbf{k x}} k^{2}}{k^{4}+4 m^{2} k_{\|}^{2}} \\
& F_{2}(\mathbf{x}) \equiv \int \frac{d^{d} k}{(2 \pi)^{d}} \frac{e^{i \mathbf{k x}}(-2 m) i k_{\|}}{k^{4}+4 m^{2} k_{\|}^{2}} .
\end{aligned}
$$

It is then helpful to realize that the integrands, without the exponential factor, are simply the convolutions of two functions, i.e.:

$$
\begin{aligned}
\frac{k^{2}}{k^{4}+4 m^{2} k_{\|}^{2}} & =\int \frac{d^{d} k^{\prime}}{(2 \pi)^{d}} F\left(\mathbf{k}^{\prime}\right) C\left(\mathbf{k}-\mathbf{k}^{\prime}\right) \\
\frac{-2 m i k_{\|}}{k^{4}+4 m^{2} k_{\|}^{2}} & =\int \frac{d^{d} k^{\prime}}{(2 \pi)^{d}} F\left(\mathbf{k}^{\prime}\right) S\left(\mathbf{k}-\mathbf{k}^{\prime}\right),
\end{aligned}
$$

where

$$
\begin{aligned}
F(\mathbf{k}) & =\frac{1}{k^{2}+m^{2}} \\
C(\mathbf{k}) & =\frac{(2 \pi)^{d}}{2} \delta\left(\mathbf{k}_{\perp}\right)\left[\delta\left(k_{\|}+i m\right)+\delta\left(k_{\|}-i m\right)\right] \\
S(\mathbf{k}) & =\frac{(2 \pi)^{d}}{2} \delta\left(\mathbf{k}_{\perp}\right)\left[\delta\left(k_{\|}+i m\right)-\delta\left(k_{\|}-i m\right)\right] .
\end{aligned}
$$

The $\delta$-functions with complex arguments should only be understood in an operational sense. The Fourier inverse transforms of these functions are easily found: 


$$
\begin{aligned}
F(\mathbf{x}) & \equiv F(r)=\frac{1}{(2 \pi)^{\frac{d}{2}}}\left(\frac{m}{r}\right)^{\frac{d-2}{2}} K_{\frac{d-2}{2}}(m r) \\
C(\mathbf{x}) & =\cosh \left(m x_{\|}\right) \\
S(\mathbf{x}) & =\sinh \left(m x_{\|}\right) .
\end{aligned}
$$

Thus, using the convolution theorem, we trivially get

$$
\begin{aligned}
& F_{1}(\mathbf{x})=\cosh \left(m x_{\|}\right) F(r) \\
& F_{2}(\mathbf{x})=\sinh \left(m x_{\|}\right) F(r) .
\end{aligned}
$$

Note that $F(r)$ is the solution of

$$
\left(-\nabla^{2}+m^{2}\right) F(r)=\delta(\mathbf{x})
$$

Then using some algebra and (A8), we can translate $\nabla \sigma \nabla$ into differentiation with respect to $x_{\|}$:

$$
\begin{aligned}
\nabla \boldsymbol{\sigma} \nabla F_{1}(\mathbf{x}) & =-\sigma_{\perp} \delta(\mathbf{x})+\sigma_{\perp} 2 m \partial_{\|}\left\{\sinh \left(m x_{\|}\right) F(r)\right\}+\left(\sigma_{\|}-\sigma_{\perp}\right) \partial_{\|}^{2}\left\{\cosh \left(m x_{\|}\right) F(r)\right\} \\
& =-\sigma_{\perp} \delta(\mathbf{x})+\sigma_{\perp} 2 m \partial_{\|} F_{2}(\mathbf{x})+\left(\sigma_{\|}-\sigma_{\perp}\right) \partial_{\|}^{2} F_{1}(\mathbf{x}) \\
\nabla \boldsymbol{\sigma} \nabla F_{2}(\mathbf{x}) & =\sigma_{\perp} 2 m \partial_{\|}\left\{\cosh \left(m x_{\|}\right) F(r)\right\}+\left(\sigma_{\|}-\sigma_{\perp}\right) \partial_{\|}^{2}\left\{\sinh \left(m x_{\|}\right) F(r)\right\} \\
& =\sigma_{\perp} 2 m \partial_{\|} F_{1}(\mathbf{x})+\left(\sigma_{\|}-\sigma_{\perp}\right) \partial_{\|}^{2} F_{2}(\mathbf{x})
\end{aligned}
$$

These forms are particularly useful when we calculate the corresponding long-distance behavior.

\section{APPENDIX B: LONG DISTANCE ASYMPTOTIC BEHAVIOR OF THE CORRELATION FUNCTIONS}

To obtain the long-distance behavior for $\boldsymbol{\nabla} \boldsymbol{\sigma} \boldsymbol{\nabla}(\mathbf{x})$, we just have to omit the first term in (A2), which is a $\delta$-function:

$$
\left.\nabla \boldsymbol{\sigma} \nabla E(\mathbf{x})\right|_{\mathbf{x} \neq \mathbf{0}}=-\left(\sigma_{\|}-\sigma_{\perp}\right) \frac{\Gamma\left(\frac{d}{2}\right)}{2 \pi^{\frac{d}{2}}} \frac{r_{\perp}^{2}-(d-1) r_{\|}^{2}}{r^{d+2}}
$$

This is the typical "FDT-violating" power law, provided that $\boldsymbol{\sigma}$ is not a simple multiple of the unit matrix. Otherwise, the amplitude of this term would be zero.

Using the "large $z$ " asymptotic expansion of the modified Besssel function [27]

$$
K_{\nu}(z) \simeq \sqrt{\frac{\pi}{2 z}} e^{-z}\left\{1+\left(\nu^{2}-\frac{1}{4}\right) \frac{1}{2 z}+\mathcal{O}\left(\frac{1}{z^{2}}\right)\right\},
$$

we can obtain the long-distance behavior for $\nabla \sigma \nabla F_{1}(\mathbf{x})$ and $\nabla \sigma \nabla F_{2}(\mathbf{x})$ as $m=$ const. $>0$ and $r \rightarrow \infty$. Due to the strong anisotropies in these functions, we consider three different scenarios:

(i.) $r_{\|}=0, r_{\perp} \rightarrow \infty$ :

Combining (A9) and the asymptotic form of $F(r)$ we find:

$$
\begin{aligned}
\left.\partial_{\|} F_{2}(\mathbf{x})\right|_{x_{\|}=0} & =\left.m F(r)\right|_{x_{\|}=0} \simeq \frac{\sqrt{\frac{\pi}{2}}}{(2 \pi)^{\frac{d}{2}}} e^{-m r_{\perp}}\left\{\left(\frac{m}{r_{\perp}}\right)^{\frac{d-1}{2}}+\mathcal{O}\left(\frac{1}{r_{\perp}^{\frac{d+1}{2}}}\right)\right\} \\
\left.\partial_{\|}^{2} F_{1}(\mathbf{x})\right|_{x_{\|}=0} & =\left.m^{2} F(r)\right|_{x_{\|}=0}+\left.\frac{1}{r} \frac{\partial F(r)}{\partial r}\right|_{x_{\|}=0} \\
& \simeq m \frac{\sqrt{\frac{\pi}{2}}}{(2 \pi)^{\frac{d}{2}}} e^{-m r_{\perp}}\left\{\left(\frac{m}{r_{\perp}}\right)^{\frac{d-1}{2}}+\mathcal{O}\left(\frac{1}{r_{\perp}^{\frac{d+1}{2}}}\right)\right\},
\end{aligned}
$$

while $\partial_{\|} F_{1}(\mathbf{x})$ and $\partial_{\|}^{2} F_{2}(\mathbf{x})$ are simply zero at $x_{\|}=0$, since they are odd functions of $x_{\|}$. Thus, finally we have 


$$
\begin{aligned}
& \left.\nabla \sigma \nabla F_{1}(\mathbf{x})\right|_{x_{\|}=0} \simeq\left(\sigma_{\perp}+\sigma_{\|}\right) m \frac{\sqrt{\frac{\pi}{2}}}{(2 \pi)^{\frac{d}{2}}} e^{-m r_{\perp}}\left\{\left(\frac{m}{r_{\perp}}\right)^{\frac{d-1}{2}}+\mathcal{O}\left(\frac{1}{r_{\perp}^{\frac{d+1}{2}}}\right)\right\} \\
& \left.\nabla \sigma \nabla F_{2}(\mathbf{x})\right|_{x_{\|}=0}=0
\end{aligned}
$$

(ii.) $r_{\|} \rightarrow \infty, \quad r_{\perp} \neq 0$ :

In addition to using the asymptotic form of $F(r)$, we can now also write $\cosh \left(m x_{\|}\right) \simeq \frac{1}{2} e^{m r_{\|}}$and $\sinh \left(m x_{\|}\right) \simeq \operatorname{sgn}\left(x_{\|}\right) \frac{1}{2} e^{m r_{\|}}$. In the following, we will keep the second leading power in $1 / r$ in order to simplify the discussion of case (iii). We find

$$
\begin{aligned}
\partial_{\|} F_{2}(\mathbf{x}) & \simeq \frac{\sqrt{\frac{\pi}{2}}}{(2 \pi)^{\frac{d}{2}}} \frac{e^{m r_{\|}}}{2} e^{-m r}\left\{\left(1-\frac{r_{\|}}{r}\right)\left(\frac{m}{r}\right)^{\frac{d-1}{2}}\right. \\
& \left.+\frac{d-1}{8 m^{2}}\left((d-3)-(d+1) \frac{r_{\|}}{r}\right)\left(\frac{m}{r}\right)^{\frac{d+1}{2}}+\mathcal{O}\left(\frac{1}{r^{\frac{d+3}{2}}}\right)\right\} \\
\partial_{\|}^{2} F_{1}(\mathbf{x}) & \simeq m \frac{\sqrt{\frac{\pi}{2}}}{(2 \pi)^{\frac{d}{2}}} \frac{e^{m r_{\|}}}{2} e^{-m r}\left\{\left(1-\frac{r_{\|}}{r}\right)^{2}\left(\frac{m}{r}\right)^{\frac{d-1}{2}}\right. \\
& \left.+\frac{d+1}{8 m^{2}}\left((d-5)-2(d-1) \frac{r_{\|}}{r}+(d+3) \frac{r_{\|}^{2}}{r^{2}}\right)\left(\frac{m}{r}\right)^{\frac{d+1}{2}}+\mathcal{O}\left(\frac{1}{r^{\frac{d+3}{2}}}\right)\right\} \\
\partial_{\|} F_{1}(\mathbf{x}) & \simeq \operatorname{sgn}\left(x_{\|}\right) \partial_{\|} F_{2}(\mathbf{x}) \\
\partial_{\|}^{2} F_{2}(\mathbf{x}) & \simeq \operatorname{sgn}\left(x_{\|}\right) \partial_{\|}^{2} F_{1}(\mathbf{x})
\end{aligned}
$$

Thus, for $r_{\perp} \neq 0$ we have in leading order:

$$
\begin{aligned}
\nabla \sigma \nabla F_{1}(\mathbf{x}) & \simeq m \frac{\sqrt{\frac{\pi}{2}}}{(2 \pi)^{\frac{d}{2}}} \frac{e^{m r_{\|}}}{2} e^{-m r}\left\{\left(\left(\sigma_{\perp}+\sigma_{\|}\right)-2 \sigma_{\|} \frac{r_{\|}}{r}+\left(\sigma_{\|}-\sigma_{\perp}\right) \frac{r_{\|}^{2}}{r^{2}}\right)\left(\frac{m}{r}\right)^{\frac{d-1}{2}}\right. \\
& \left.+\mathcal{O}\left(\frac{1}{r^{\frac{d+1}{2}}}\right)\right\} \\
\nabla \boldsymbol{\sigma} \nabla F_{2}(\mathbf{x}) & \simeq \operatorname{sgn}\left(x_{\|}\right) \nabla \sigma \nabla F_{1}(\mathbf{x}) .
\end{aligned}
$$

(iii.) $r_{\|} \rightarrow \infty, \quad r_{\perp}=0$ :

Note that (B5) was obtained exploiting only $r_{\|} \rightarrow \infty$. Setting $r_{\perp}=0$ has two important consequences: since now $r=r_{\|}$, the exponential decays cancel and, further, the amplitude of the $(1 / r)^{\frac{d-1}{2}}$ term will vanish. Invoking the next-to-leading terms in (B5) yields:

$$
\begin{aligned}
& \left.\nabla \sigma \nabla F_{1}(\mathbf{x})\right|_{r_{\perp}=0} \simeq-\sigma_{\perp} \frac{\sqrt{\frac{\pi}{2}}}{(2 \pi)^{\frac{d}{2}}}\left\{\frac{d-1}{2 m}\left(\frac{m}{r_{\|}}\right)^{\frac{d+1}{2}}+\mathcal{O}\left(\frac{1}{r_{\|}^{\frac{d+3}{2}}}\right)\right\} \\
& \left.\left.\nabla \sigma \nabla F_{2}(\mathbf{x})\right|_{r_{\perp}=0} \simeq \operatorname{sgn}\left(x_{\|}\right) \nabla \sigma \nabla F_{1}(\mathbf{x})\right|_{r_{\perp}=0} .
\end{aligned}
$$

which are the desired results.

[1] D. Beysens and M. Gbadamassi, Phys. Rev. A22 (1980) 2250; H. Kiefte, M.J. Clouter and R. Penney, Phys. Rev. B30 (1984) 4017; B.M. Law, P.N. Segrè, R.W. Gammon and J.V. Sengers, Phys. Rev. A41 (1990) 816; P.N. Segrè, R.W. Gammon, J.V. Sengers and B.M. Law, Phys. Rev. A45 (1992) 714; P.N Segrè, R. Schmitz and J.V Sengers, Physica A195 (1993) 31.

[2] S. Katz, J.L. Lebowitz and H. Spohn, Phys. Rev. B28 (1983) 1655; J. Stat. Phys. 34 (1984) 497.

[3] B. Schmittmann and R.K.P. Zia, in: Phase Transitions and Critical Phenomena Vol. 17, eds. C. Domb and J.L. Lebowitz, (Academic Press, N.Y., 1995).

[4] R. Kubo, Rep. Progr. Phys. 29 (1966) 255. 
[5] R.K.P. Zia, K. Hwang, B. Schmittmann and K.-t Leung, Physica A194 (1993) 183.

[6] J.C. Dainty, Laser Speckle and Related Phenomena (Springer, Berlin, $2^{\text {nd }}$ edition, 1984).

[7] M. Blume, V.J. Emery and R.B. Griffiths, Phys. Rev. A4 (1971) 1071.

[8] R.B. Potts, Proc. Camb. Phil. Soc. 48 (1952) 106; F.Y. Wu, Rev. Mod. Phys. 54 (1982) 235.

[9] See e.g., S. Chandra, Superionic Solids. Principles and Applications (North Holland, Amsterdam 1981).

[10] M. Aertsens and J. Naudts, J. Stat. Phys. 62 (1990) 609.

[11] M. Rubinstein, Phys.Rev. Lett. 59 (1987) 1946; T.A.J. Duke, Phys. Rev. Lett. 62 (1989) 2877; Y. Schnidman, in: Mathematics in Industrial Problems IV, ed. A. Friedman (Springer, Berlin 1991); B. Widom, J.L. Viovy and A.D. Desfontaines, J. Phys I (France) 1 (1991) 1759.

[12] O. Biham, A.A. Middleton, and D. Levine, Phys. Rev. A46 (1992) R6128; K.-t. Leung, Phys. Rev. Lett. 73 (1994) 2386.

[13] B. Schmittmann, K. Hwang and R.K.P. Zia, Europhys. Lett. 19 (1992) 19.

[14] I. Vilfan, R.K.P. Zia and B. Schmittmann, Phys. Rev. Lett. 73 (1994) 2071.

[15] D.P. Foster, C. Godrèche, J. Stat. Phys. 76 (1994) 1129.

[16] G. Korniss, B. Schmittmann and R.K.P. Zia, Europhys. Lett. 32 (1995) 49 and J. Stat. Phys. 86 (1997) 721.

[17] M.R. Evans, D.P. Foster, C. Godrèche and D. Mukamel, Phys. Rev. Lett. 78 (1995) 208 and J. Stat. Phys. 80 (1995) 69.

[18] C. Godrèche and S. Sandow, private communications, to be submitted.

[19] G. Korniss, B. Schmittmann and R.K.P. Zia, Physica A239 (1997) 111.

[20] G. Korniss, B. Schmittmann and R.K.P. Zia, J. Phys. A30 (1997) 3837.

[21] F. Spitzer, Adv. Math. 5 (1970) 246.

[22] N. van Kampen, Adv. Chem. Phys. 34 (1976) 245.

[23] G. Korniss, Ph.D. Dissertation. 102 pp., Virginia Polytechnic Institute and State University, April 1997.

[24] H.K. Janssen and B. Schmittmann, Z. Phys. B64 (1986) 503; K.-t Leung and J.L.Cardy, J. Stat. Phys. 44 (1986) 567 and (1986) 1087.

[25] M.Q. Zhang, J.-S. Wang, J.L Lebowitz and J.L. Vallés, J. Stat. Phys. 52 (1988) 1461.

[26] M.S. Rudzinsky and R.K.P. Zia, J. Phys. A29 (1996) 6717.

[27] I.S. Gradshteyn and I.M. Ryzhik, Table of Integrals, Series and Products (Academic Press, N.Y., 1994). 
(a)

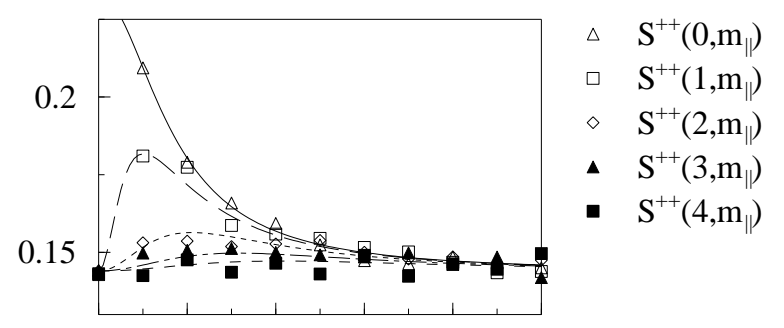

(b)

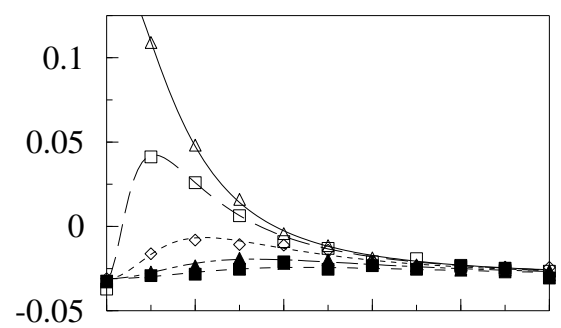

$$
\begin{array}{cl}
\triangle & \operatorname{Re}\left\{\mathrm{S}^{+-}\left(0, \mathrm{~m}_{\|}\right)\right\} \\
\square & \operatorname{Re}\left\{\mathrm{S}^{+-}\left(1, \mathrm{~m}_{\|}\right)\right\} \\
\diamond & \operatorname{Re}\left\{\mathrm{S}^{+-}\left(2, \mathrm{~m}_{\|}\right)\right\} \\
\Delta & \operatorname{Re}\left\{\mathrm{S}^{+-}\left(3, \mathrm{~m}_{\|}\right)\right\} \\
- & \operatorname{Re}\left\{\mathrm{S}^{+-}\left(4, \mathrm{~m}_{\|}\right)\right\}
\end{array}
$$

(c)

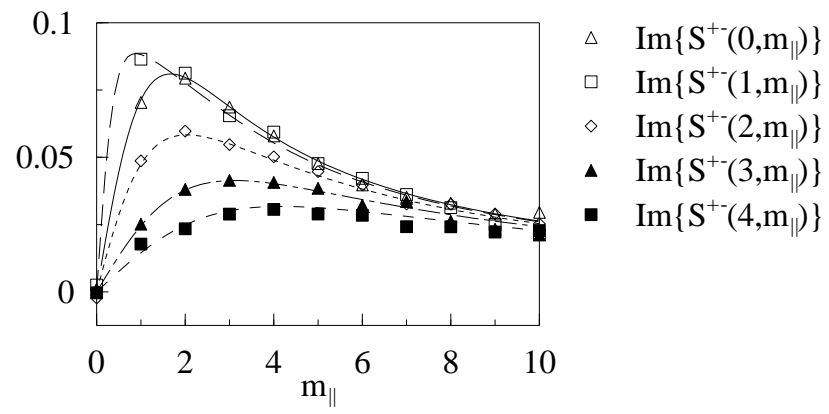

FIG. 1. Steady state structure factors (a) $S^{++}(\mathbf{k})$, (b) $\operatorname{Re}\left\{S^{+-}(\mathbf{k})\right\}$, (c) $\operatorname{Im}\left\{S^{+-}\right.$(k) $\}$for an $L=100$ system at $\gamma=0.02$, $E=0.279$ and $\bar{\rho}=0.175$. Structure factors are plotted against the integer $m_{\|}=\frac{k_{\|} L}{2 \pi}$, while $m_{\perp}=\frac{k_{\perp} L}{2 \pi}$ is taken as a parameter. Lines are representing the fitted theoretical curves. 
(a)

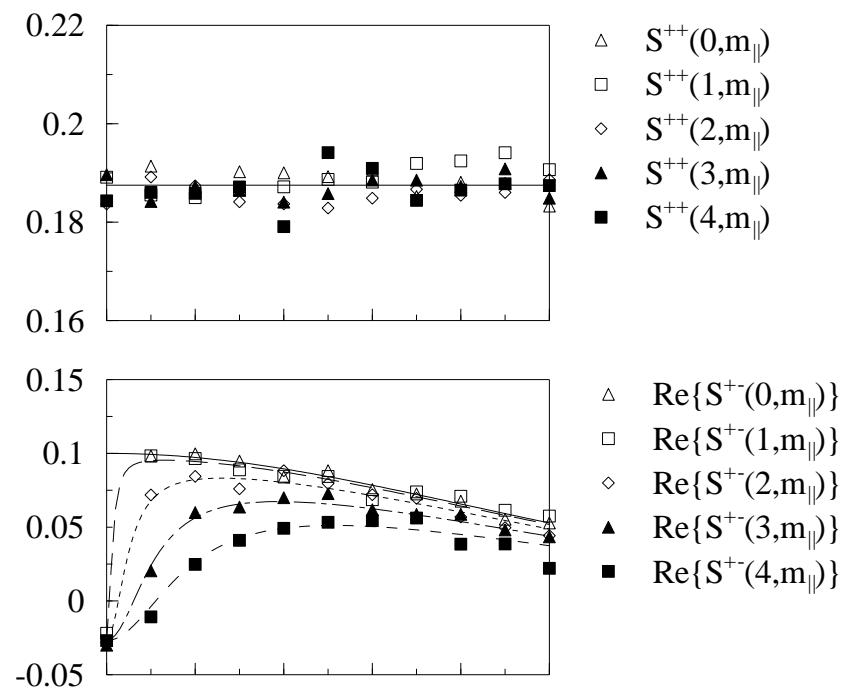

(b)

(c)

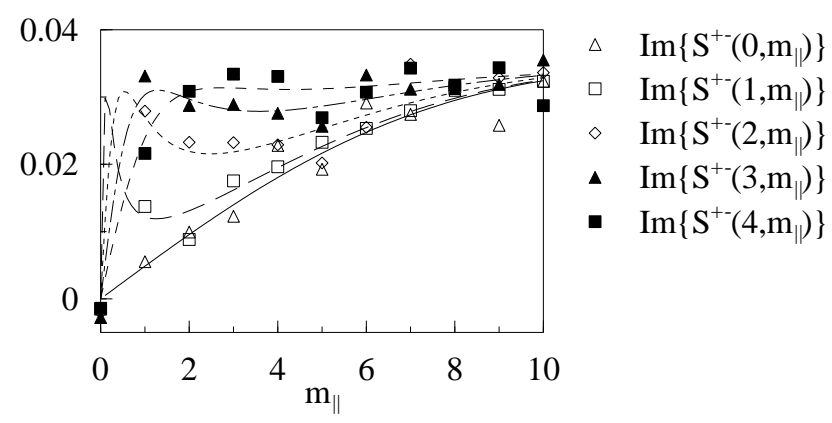

FIG. 2. Steady state structure factors (a) $S^{++}(\mathbf{k})$, (b) $\operatorname{Re}\left\{S^{+-}(\mathbf{k})\right\}$, (c) $\operatorname{Im}\left\{S^{+-}\right.$(k) $\}$for an $L=100$ system at $\gamma=1.00$, $E=\infty$ and $\bar{\rho}=0.25$. Structure factors are plotted against the integer $m_{\|}=\frac{k_{\|} L}{2 \pi}$, while $m_{\perp}=\frac{k_{\perp} L}{2 \pi}$ is taken as a parameter. Lines are representing the fitted theoretical curves. 

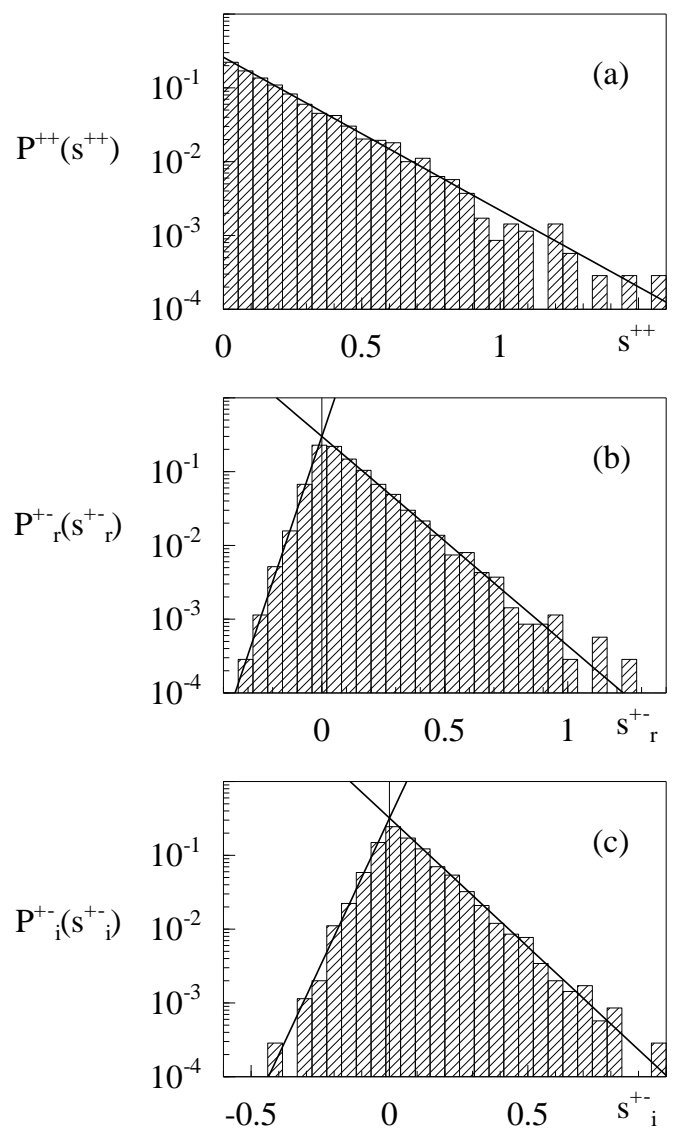

FIG. 3. Histograms representing the distributions of the $\mathbf{k}=\frac{2 \pi}{L}(0,1)$ structure factors for (a) $\frac{n_{\mathbf{k}}^{+} n_{-\mathbf{k}}^{+}}{V}$, (b) $\frac{\operatorname{Re}\left[n_{\mathbf{k}}^{+} n_{-\mathbf{k}}^{-}\right]}{V}$ and (c) $\frac{\operatorname{Im}\left[n_{\mathbf{k}}^{+} n_{-\mathbf{k}}^{-}\right]}{V} . L=100, \gamma=0.02, E=0.279$ and $\bar{\rho}=0.175$. Theoretical distributions (a) $P^{++}\left(s^{++} ; \mathbf{k}\right),(\mathrm{b}) P_{r}^{+-}\left(s_{r}^{+-} ; \mathbf{k}\right)$ and $(\mathrm{c})$ $P_{i}^{+-}\left(s_{i}^{+-} ; \mathbf{k}\right)$ are plotted with solid lines on the same graphs. 

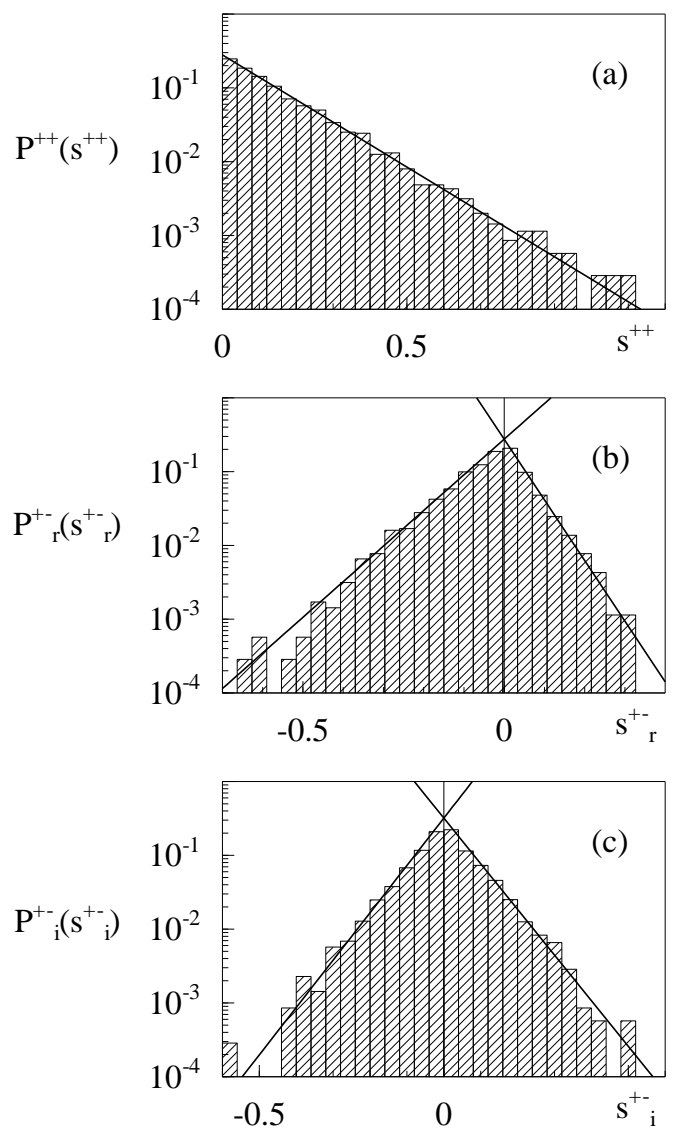

FIG. 4. Histograms representing the distributions of the $\mathbf{k}=\frac{2 \pi}{L}(1,0)$ structure factors for (a) $\frac{n_{\mathbf{k}}^{+} n_{-\mathbf{k}}^{+}}{V}$, (b) $\frac{\operatorname{Re}\left[n_{\mathbf{k}}^{+} n_{-\mathbf{k}}^{-}\right]}{V}$ and (c) $\frac{\operatorname{Im}\left[n_{\mathbf{k}}^{+} n_{-\mathbf{k}}^{-}\right]}{V} . L=100, \gamma=0.02, E=0.279$ and $\bar{\rho}=0.175$. Theoretical distributions (a) $P^{++}\left(s^{++} ; \mathbf{k}\right),(\mathrm{b}) P_{r}^{+-}\left(s_{r}^{+-} ; \mathbf{k}\right)$ and $(\mathrm{c})$ $P_{i}^{+-}\left(s_{i}^{+-} ; \mathbf{k}\right)$ are plotted with solid lines on the same graphs. 